\title{
Functional endoscopy in neurogenic dysphagia: a feasibility study focusing on the esophageal phase of swallowing $\square$
}

\section{다 (1) $\odot$}

Authors

Jan Rückert ${ }^{1}$, Philipp Lenz ${ }^{2,3}$, Hauke Heinzow ${ }^{2}$, Johannes Wessling ${ }^{4,5}$, Tobias Warnecke ${ }^{6}$, Ingo F. Herrmann ${ }^{7}$, Michael Strahl7, Frank Lenze ${ }^{1}$, Tobias Nowacki ${ }^{1,2}$, Dirk Domagk ${ }^{1,2}$

Institutions

1 Department of Medicine I, Josephs-Hospital Warendorf, Academic Teaching Hospital, University of Muenster, Warendorf, Germany

2 Department of Medicine B, University of Muenster, Muenster, Germany

3 Department of Palliative Medicine, University of Muenster, Muenster, Germany

4 Department of Clinical Radiology, University of Muenster, Muenster, Germany

5 Department of Radiology, Clemens Hospital Muenster, Muenster, Germany

6 Department of Neurology, University of Muenster, Muenster, Germany

7 Reflux Center Duesseldorf, ENT, Duesseldorf, Germany

submitted 17.8 .2020

accepted after revision 30.12 .2020

\section{Bibliography}

Endosc Int Open 2021; 09: E646-E652

DOI 10.1055/a-1380-3224

ISSN 2364-3722

(C) 2021. The Author(s).

This is an open access article published by Thieme under the terms of the Creative Commons Attribution-NonDerivative-NonCommercial License, permitting copying and reproduction so long as the original work is given appropriate credit. Contents may not be used for commercial purposes, or adapted, remixed, transformed or built upon. (https://creativecommons.org/licenses/by-nc-nd/4.0/)

Georg Thieme Verlag KG, Rüdigerstraße 14,

70469 Stuttgart, Germany

\section{Corresponding author}

Dirk Domagk, MD FASGE, Department of Medicine I, JosephsHospital Warendorf, Academic Teaching Hospital University of Muenster, Am Krankenhaus 2, 48231 Warendorf, Germany Fax: +49 2581 - 201402

domagkd@uni-muenster.de

Supplementary material is available under

https://doi.org/10.1055/a-1380-3224

\section{ABSTRACT}

Background and study aims Due to demographic transition, neurogenic dysphagia has become an increasingly recognized problem. Patients suffering from dysphagia often get caught between different clinical disciplines. In this study, we implemented a defined examination protocol for evaluating the whole swallowing process by functional endoscopy. Special focus was put on the esophageal phase of swallowing.

Patients and methods This prospective observational multidisciplinary study evaluated 31 consecutive patients with suspected neurogenic dysphagia by transnasal access applying an ultrathin video endoscope. Thirty-one patients with gastroesophageal reflux symptoms were used as a control group. We applied a modified approach including standardized endoscopic positions to compare our findings with fiberoptic endoscopic evaluation of swallowing and high-resolution manometry. The primary outcome measure was feasibility of functional endoscopy. Secondary outcome measures were adverse events (AEs), tolerability, and pathologic endoscopic findings.

Results Functional endoscopy was successfully performed in all patients. No AEs were recorded. A variety of disorders were documented by functional endoscopy: incomplete or delayed closure of the upper esophageal sphincter in retroflex view, clearance disturbance of tubular esophagus, esophageal hyperperistalsis, and hypomotility. Analysis of results obtained with the diagnostic tools showed some discrepancies.

Conclusions By interdisciplinary cooperation with additional assessment of the esophageal phase of deglutition using the innovative method of functional endoscopy, the diagnosis of neurogenic disorders including dysphagia may be significantly improved, leading to a better clinical understanding of complex dysfunctional patterns. To the best of our knowledge, this is the first study to show that a retroflex view of the ultrathin video endoscope within the esophagus can be safely performed. [NCT01995929] 


\section{Introduction}

Swallowing is the process used to transport food from the mouth to the stomach. Thus, normal swallowing is an essential and complex mechanism that requires the integration of processes controlled by the central and peripheral nervous system, the coordinated interaction of more than 25 pairs of muscles as well as intact anatomical structures of the upper aerodigestive tract and esophagus [1]. Typically, swallowing can be divided into four stages: 1. oral preparation (i.e., salivating, masticating, formation of a bolus of suitable size and consistency, sealed oral cavity by tongue and soft palate to prevent leaking of food into the oropharynx); 2. oral propulsion (i. e., transfer of the bolus through the mouth using the tongue while the swallowing reflex is triggered at the end of this stage) 3. pharyngeal (i. e., reflex activities in the pharyngeal passage to protect the airway and moving the food bolus to upper esophageal sphincter [UES]); and 4. esophageal (i. e., tubular structure with a peristalsis wave that is regulated by the autonomous nervous system and is divided into two different parts: relaxation and contraction aiming to carry the bolus to the lower esophageal sphincter (LES) and stomach) [2]. Both structural and functional abnormalities can lead to impaired swallowing and a wide variety of diseases and disorders can cause dysphagia, e. g. Parkinson's disease (PD), dementia, atypical parkinsonism (APS), ischaemic or haemorrhagic stroke, brain injury, achalasia, diabetic neuropathy or myasthenia gravis [3-8]. Neurological disorders are the most common cause for dysphagia. Especially with regard to the demographic change in western countries, neurogenic dysphagia is a widely spread disorder. Approximately 5 million people suffer from dysphagia in Germany and $50 \%$ of all neurological and geriatric patients are affected by neurogenic dysphagia [8], which mainly affects the oropharyngeal phase of swallowing [9].

Adequately diagnosing and monitoring the course or therapeutic effects of dysphagia in neurological patients is a challenge. Sophisticated diagnostic tools are important to identify underlying mechanisms of swallowing disorders which is essential to provide effective, causative treatment. These diagnostic challenges are further complicated by the fact that multiple methods are needed to evaluate all phases of swallowing and moreover, patients suffering from dysphagia are often treated in various clinical disciplines, i. e. geriatric units while others are treated in cardiac or internal wards [10]. This dilemma might be due to a lack of pathophysiological knowledge and an inability of the existing diagnostic tools to directly visualize all phases of deglutition within one procedure. To identify oropharyngeal dysphagia, fiberoptic endoscopic evaluation of swallowing (FEES) is a well-established diagnostic tool [11]. Originally, it was developed to identify disorders within the pharyngeal phase of swallowing (i.e. aspiration, penetration, residues). Therefore, a flexible laryngoscope is introduced via the nasal cavity to examine the anatomy and movement patterns of the oral cavity, pharynx, and larynx while the patient swallows food of different consistencies [1,3]. Furthermore, high-resolution manometry (HR-manometry) can be used to evaluate disorders in the esophageal phase of swallowing. While its diagnostic ac- curacy corresponds to the number of pressure sensors that are placed in the esophagus, contractility can be monitored over time and results can be visualized in pressure topography plots [12].

Recent studies indicate that functional disorders of the esophagus and the UES are often asymptomatic and remain undetected [1]. Moreover, disturbances in the pharyngeal phase of swallowing can impair opening of the UES and vice versa. More precisely, this can lead to retention of food in the hypopharynx region with subsequent risks of retention in the piriform sinuses, penetration or aspiration [2]. Thus, the aim of this study was to test the feasilbility and to develop an examination protocol for functional endoscopy in patients with neurogenic dysphagia, which can be used to directly visualize and evaluate all phases of the swallowing process within one examination. This approach may fill the diagnostic gap between the existing techniques and subsequently improve the understanding of the underlying pathophysiological mechanisms in neurogenic dysphagia. Notably, the European Society for Swallowing Disorders recommends a multidimensional swallowing assessment to provide complementary information on the different stages of the swallowing procedure [13]. In the present study, especially the esophageal phase of deglutition, particularly the function of the UES, tubular esophagus and LES in retroflex vision was evaluated focussing on feasibility, safety, and tolerability of the procedure as outcome measures. Furthermore, we added a comparison group of patients with gastroesphageal reflux symptoms and dysphagia to evaluate differences in endoscopic findings with regard to swallowing function [14].

\section{Patients and methods}

This prospective observational study was performed in a multidisciplinary setting: From December 2013 to October 2017, 31 consecutive patients were examined by functional endoscopy applying an ultrathin video endoscope. Diagnostic workup was completed by HR-manometry and FEES. All patients with suspected neurogenic dysphagia were considered eligible for the study. Prior to inclusion, organic abnormalities were excluded by upper gastrointestinal endoscopy. Exclusion criteria were age $<18$, the inability to understand information for participation or refusal to participate. This study conformed to the ethical guidelines of the 1975 Declaration of Helsinki and its later amendments and was approved by the appropriate local Ethics Committee of Muenster, Germany (AZ 2010-214-f-S). The anonymity of patients enrolled in the study was carefully protected and investigations reported in the manuscript were performed with written informed consent. The primary outcome measure was feasibility of the functional endoscopy. Secondary outcome measures were adverse events (AEs) within 1 week to quantify safety and tolerability. Furthermore, obtained results were compared with HR-manometry results. The study was registered at ClinicalTrials.gov (NCT01995929). In addition, we examined 31 patients with gastroesphageal reflux symptoms and dysphagia as a control group that included patients without any neurological disorders. 


\section{Functional endoscopy}

For this technique, an ultrathin flexible video endoscope (BF3 C160, Olympus Europe) with the corresponding video processor (EVIS EXERA III CV-190) was used. The endoscope has an outer diameter of $3.8 \mathrm{~mm}$, a working length of $60 \mathrm{~cm}$, and an angulation range up to $180^{\circ}$. Each examination was documented as a video sequence for later analysis by an interdisciplinary team. Standardized endoscopic positions (TableS1) were defined in analogy to functional pharyngoesophagoscopy [15] and video panendoscopy [16] to ensure interprocedural comparability. For examination, patients were in a sitting position and unsedated. To allow an undisturbed swallowing act, transnasal access was selected without using sedatives or local anesthetics. Decongestant nose drops were used to facilitate introduction of the scope and to avoid irritation of the mucosa. The scope was introduced via the inferior meatus of the nose and passed through the nasal and oral cavity, pharynx, and esophagus carefully towards the LES. Upon reaching the lower esophagus, typically at position 5 , the actual analysis commenced. At that point, the patient was asked to perform at least three dry swallows to obtain an overview of the present function. Subsequently, the patient swallowed food of three different consistencies: liquid, semi-solid, and solid (water, yogurt and bread). Then, the biomechanical movement of the structures that were to be investigated at every position mentioned in Table S1 were assessed in real-time visualization while the patient swallowed the different types of food. It was necessary to do some water swallows from time to time to clean the structures from the previous swallow. All positions were evaluated consecutively. The required number of performed swallows depended on patient cooperativity and the recorded image quality of the moving structures. For this reason, the length of the examination varied between 15 and 30 minutes. Targeted communication during the procedure was important to improve the quality of diagnosis and enhance patient acceptance. After every examination, an image by image analysis was done. The results were structured in three parts: UES, tubular esophagus, and LES. All detected pathologies were registered and categorized to the relevant part. Because of the lack of established scoring systems or validated criteria for measuring and evaluating the esophageal phase with endoscopic methods, the previously established severity score established by Warnecke et al. to assess neurogenic dysphagia in FEES studies was adapted and modified to four defined severity levels (normal, mild, moderate and severe) for later comparisons. Depending on the intensity of the documented pathologies and the clinical signs of the patient before and during the procedure, an interdisciplinary panel of experts analyzed all video sequences. Patients were monitored as inpatients or outpatients for 1 week after the procedure and were instructed to inform the investigator about any discomfort, hematemesis, or signs of inflammation.

\section{Fiberoptic endoscopic evaluation of swallowing}

This method was originally described in 1988 by Langmore et al. [11]. Today it is a well-established diagnostic tool for patients with dysphagia, especially to evaluate the oropharyngeal phase of swallowing. In our multidisciplinary team, FEES was done by the standard FEES protocol described elsewhere [17]. As mentioned above, the results were documented and classified into the four severity levels (normal, mild, moderate, and severe).

\section{HR-manometry}

HR-manometry has widely replaced conventional manometry [18] because it allows acquisition of pressure conditions of the esophagus in a high spatial resolution [19] due to an increased number of pressure sensors, leading to a decreased distance between each sensor, usually $10 \mathrm{~mm}$ in between [20]. An additional benefit of HR-manometry is the fixed position of the catheter, which leads to fewer artifacts, more information about clinically unnoticed problems, and examiner-independent results [19]. Furthermore, HR-manometry can yield pressure topography plots, i.e. high-resolution esophageal pressure topography (HREPT), which enables a more intuitive understanding of the entire motility function of the esophagus $[18,21]$. In this study, we used the ManoScan 360 EG System with the corresponding high-resolution ManoScan esophagus catheter with 36 sensors, 10 -mm spacing between each sensor, and a diameter of $4.2 \mathrm{~mm}$. The computer-based algorithm Sierra adult version (Sierra Scientific Instruments, Los Angeles, California, United States), was used for data collection and analysis [22, 23]. For examination, every patient was in a half-sitting position $\left(45^{\circ}\right)$ and awake. After placing the probe in the esophagus we performed an adjusted standard protocol to investigate the swallowing function [19]. The procedure included 10 swallows with $5 \mathrm{~mL}$ of water and a 30 -second rest between each swallow to allow complete relaxation of the esophagus. Results were evaluated and structured into three parts: UES, tubular esophagus, and LES, starting with the evaluation of the LES because dysfunctions in this area can impair peristalsis of the whole esophagus. Findings were categorized as minor or major disorders of esophageal peristalsis according to previous HR-manometry studies, e. g. the Chicago Classification Version 3.0 [24-26]. In a second step, disorders were further allocated to four severity categories (normal, mild, moderate and severe) by assessing established values for evaluation of the LES (Integrated Relaxation Pressure (IRP, [mmHg]), esophageal motility (Distal Contractile Integral $\left.(\mathrm{DCl})\left[\mathrm{mmHg}^{*} \mathrm{~s}^{*} \mathrm{~cm}\right]\right)$, Contractile Front Velocity (CFV) [cm/s]), Intrabolus Pressure (IBP) $[\mathrm{mmHg}]$ and Distal Latency (DL) and UES (Mean basal pressure) $[\mathrm{mmHg}]$ and Relaxation duration [ms]) [27]. Based on the degree of deviation from established normal values, results were categorized by two independent experienced investigators (DD and IFH).

\section{Statistics}

Descriptive statistics, tables, and diagrams to describe the patient characteristics were created by using Microsoft Excel and Word, respectively. Cohen's kappa analyses were performed to compare the findings of the competing diagnostics. The analyses were executed using IBM SPSS Statistics Version 20 (Armonk, New York, United States: IBM Corp.). 


\section{Results}

Thirty-one patients ( 23 men, 8 women) in the group with suspected neurogenic dysphagia were included. The mean age of all patients was 62.9 years, \pm 13.1 and height average was $173.1 \mathrm{~cm}, \pm 8.3$. For reasons of clarity, the subjects were divided into three groups based on the main diagnosis: PD $(n=11)$, APS $(n=7)$ and others $(n=13)$. Diseases in the APS group were multiple system atrophy and progressive supranuclear palsy. Other diseases included both known and unknown disorders: congenital or acquired achalasia, myasthenia gravis, polyneuropathy, oculopharyngeal muscular dystrophy, diabetic neuropathy, and dysphagia of unknown origin. Overall 31 patients were examined by functional endoscopy, 21 of whom underwent HR-manometry and consecutively 19 underwent FEES. Baseline patient characteristics are depicted in TableS2. To quantify the severity of PD, modified Hoehn and Yahr stages were also documented [28]. This leds to the distribution in stadium $2(n=4), 3(n=3), 4(n=2)$ and $5(n=2)$.

The primary outcome measure was the feasibility of the functional endoscopy. All investigated patients were successfully examined. Transnasal access was practicable in all cases. Retroflection of the endoscope within the esophagus could be performed securely without AE during the examination or follow up. Safety and tolerability were part of the secondary outcome measures. No AEs were reported.

All included patients were examined by functional endoscopy. Depending on the swallowing phase, a substantial difference between the documented severity levels was found (TableS3). The most affected part was the tubular esophagus in the esophageal phase of swallowing. Notably, dysfunctions in the region of LES were found very rarely.

During the procedure, a variety of yet unclassified disorders were documented in functional endoscopy: Incomplete or delayed closure of the UES and LES, hyper- or hypotonicity of the UES and LES ( $\triangleright$ Video 1 ), clearance disturbance of tubular esophagus because of esophageal hyperperistalsis or hypomotility, collapsing esophagus leading to aspiration ( $\triangleright$ Video $2 \mathbf{a}, \mathbf{b}$ ). Especially, patients with APS frequently showed clearance disorders in the tubular esophagus (71\%) (Table S4). In comparison to that, only $36 \%$ of the patients with PD showed a deficit in this area. In patients suffering from other related diseases, we observed dysfunctions of the tubular esophagus in $62 \%$. The results came out similar to the tests of the UES function: Just $18 \%$ of the patients with PD had dysfunction, whereas $43 \%$ in APS and $38 \%$ of the others were affected. As mentioned previously, malfunctions in the region of the LES are detected rarely and the number of patients affected is negligible.

The evaluation of the pharyngeal phase of deglutition at P1 ( $\triangleright$ Video 3 ) with both endoscopic techniques (functional endoscopy and FEES) showed discrepant results (Fig. S1). With FEES, we categorized six patients as normal, whereas six others were mildly impaired. In contrast, functional endoscopy classified nine patients as normal and just one patient as mild. Regarding moderate and severe restrictions, both techniques yielded comparable results (functional endoscopy: 6 moderate, 2 severe; FEES: 3 moderate, 3 severe).

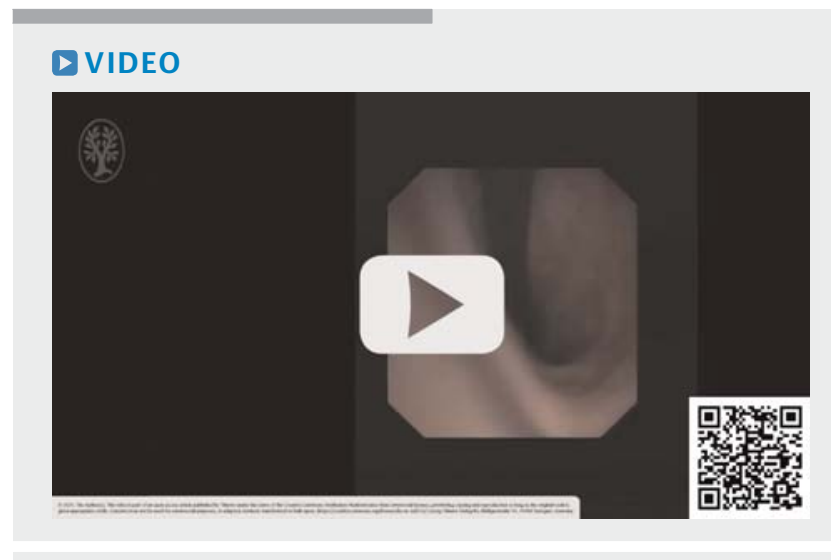

Video 1 Functional endoscopy of increased and prolonged tonus of upper esophageal sphincter.

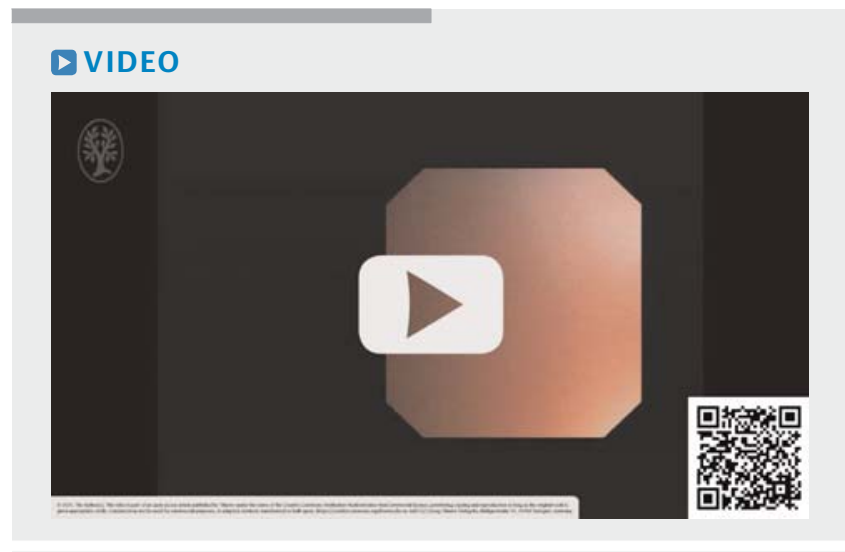

- Video 2a Functional endoscopy showing a collapsing esophagus.

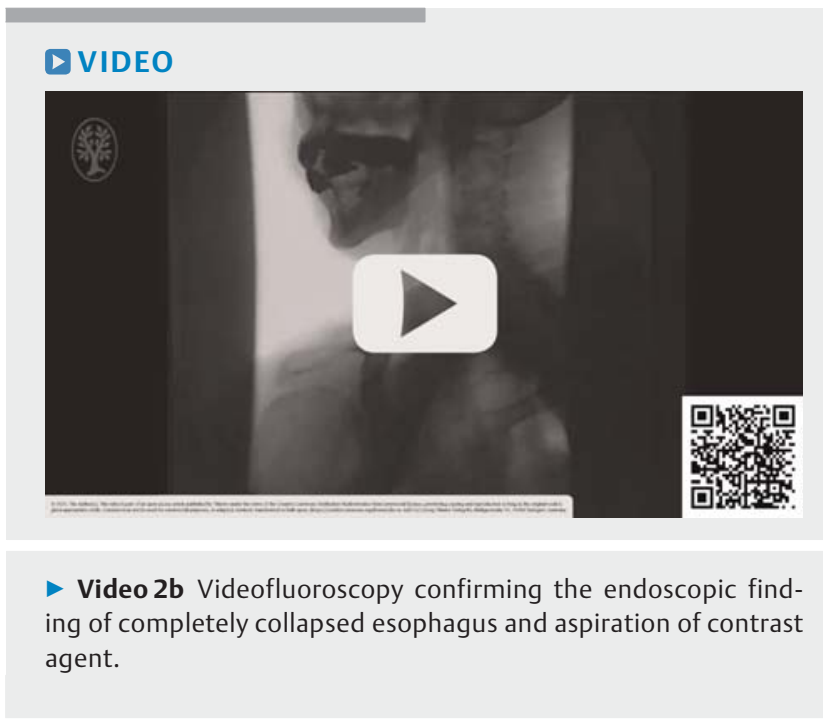

Patients who were evaluated with functional endoscopy and HR-manometry were compared according to the results of the assessment of the UES (Fig. S2). Functional endoscopy classified 15 patients as normal, two as mild, three as moderate and 


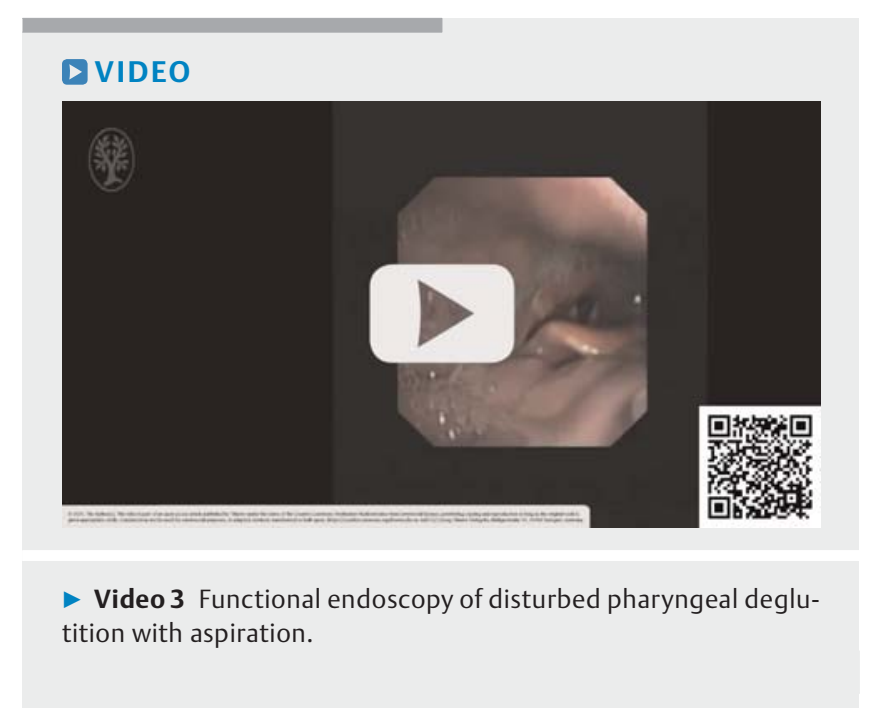

one as severe. In comparison to that HR-manometry divided 10 subjects as normal, seven as mild, two as moderate, and two as severe. Cohen's kappa coefficient is 0.141 , SE of kappa is 0.136 and the $95 \%$ confidence interval lies between -0.125 and 0.407 .

Consequently, we compared the results of functional endoscopy and HR-manometry assessing the function of the tubular esophagus (Fig.S3). Functional endoscopy detected normal swallows in six cases, four mild, six moderate, and in five cases of severe restrictions. HR-manometry determined normal swallows in two cases, 12 mild, five moderate, and two severe impairments. Cohen's kappa coefficient was 0.323, SE of kappa 0.123 and the $95 \%$ confidence interval lay between 0.082 and 0.563 .

Furthermore, we assessed the function of the lower esophagus in direct comparison of functional endoscopy and HRmanometry (Fig.S4). Both diagnostics yielded similar results (functional endoscopy: normal 21 , mild 0 , moderate 0 , severe 0 ; HR-manometry: normal 17, mild 4, moderate 0 , severe 0 ). For this reason, Cohen's kappa coefficient cannot be determined since there was no difference found.

Moreover, we analysed the relationship between the function of the pharyngeal phase and the UES (Fig. S5). Overall, the results suggest considerable agreement, especially in the group with a normal swallowing function (P1: 20 vs. P2/P3: 21). In patients with mild (P1: 3 vs. P2/P3: 5), moderate (P1: 6 vs. P2/P3: 4) and severe restrictions (P1: 2 vs. P2/P3: 1), disparities are noticeable.

In addition, we examined 31 patients with gastroesphageal reflux symptoms and dysphagia (TableS5) who served as control group without suspected neurogenic disorder. Mean age of all patients was 48.4 years, \pm 15.4 , around $65 \%$ of the comparison group were female. In analogy to results obtained from the patients with neurogenic dysphagia, the esophageal phase of swallowing was examined by functional endoscopy. Dysfunction of the LES was more frequent with around $77 \%$ of the patients showing insufficiency. With regard to the tubular esophagus, we frequently monitored gas reflux (22 patients, $71 \%$ ), and in four patients, also bile reflux. Insufficiency of the UES was only observed in one of 31 patients ( $3 \%$ of the cases).

\section{Discussion}

The current study was able to show that functional endoscopy is a safe and effective method to evaluate the whole swallowing process within one procedure in patients suffering from suspected neurogenic dysphagia. None of the investigated patients showed adverse side effects or complications such as hematemesis or signs of inflammation during or after the examination. The assessment of the esophageal phase of swallowing with a direct view of the involved structures was of specific interest for our new approach. Positions 3, 4, and 6 (Table S1) with the retrograde view within the esophagus, in particular, represent novel opportunities to enhance the understanding of underlying physiological and pathological conditions in the esophagus. We could show that retroflection can be performed securely in the upper and lower esophagus. This novel diagnostic approach with the main focus on retroflex endoscopic inspection reveals a substantial proportion of dysmotility disorders within the tubular esophagus and UES that manometric studies may fail to detect. This feature leads to an improvement in examination quality and may yield a substantial amount of useful knowledge and better understanding of pathophysiology ( $\vee$ Videos 1,2 , and 3 ). Compared to conventionally employed diagnostic approaches to study motility disorders, such as FEES and HR-manometry, functional endoscopy enables evaluation of the entire swallowing process.

\section{Documented pathologies in the esophageal phase of swallowing}

We documented a variety of disorders in evaluation of the esophagus (TableS4). With regard to the evaluation strategy in HR-manometry studies based on the current Chicago Classification [24,29], we divided the esophagus into three sections to be investigated: LES, tubular esophagus, and UES, whereby the latter is still not part of the Chicago Classification but may be monitored by HR-manometry [30,31]. Frequently, the underlying cause for swallowing disorders is based on motility dysfunction, such as hypertonicity and hypotonicity in respective regions. This leads to impaired transport of food through the esophagus [2]. In addition, disorders in the esophageal phase and pharyngeal phase of swallowing can mutually influence each other [2]. Thus, we analyzed the swallowing function at P1 and P2/P3 (Fig. S5). The results suggest that dysfunctions in one of the target areas often interfere with the neighboring regions. Yet it is challenging to determine the underlying disorder of an impaired swallowing phase because of the interdependence between the phases [2]. At this point, the various perspectives of functional endoscopy using the antegrade and retrograde view allow a better allocation of disorders to a certain region in the upper gastrointestinal tract.

Although some studies suggest that the pharyngeal phase is significantly more often affected than esophageal phase in patients suffering from neurogenic dysphagia, our data indicate a different view [9]. Despite the limited study size, our data sug- 
gest that disturbances in the tubular esophagus are more frequent, possibly due to an increased or decreased motility function in the oropharynx. A possible explanation for this might be the heterogeneity of our study group: we investigated 11 patients with PD, seven patients with APS, and 13 patients with other diseases, as previously mentioned. With regard to the pathologic findings in patients suffering from PD or APS, the latter patients more often had dysfunctions in the esophageal phase than did the patients with PD. This is also in line with findings in other studies, which demonstrated that severe dysphagia occurs earlier and more frequently in the course of the disease [32]. In the control group including patients with gastroesophageal reflux symptoms, motility disorders are frequently found in the LES, whereas both tubular esophagus (apart from endoscopically documented gas and bile reflux) and UES are rarely affected (Table S5).

\section{Challenging problems in the evaluation and determining the severity levels in comparison with the other diagnostic tools}

For a successful investigation of the swallowing function, it is essential to position the endoscope at all previously defined sections (Table S1). The results can be interpreted during and after the procedure using video analysis. In order to ensure comparability of our results with established diagnostic methods, we defined four severity levels for the esophageal phase of swallowing according to a previously published classification by Warnecke et al. for neurogenic dysphagia concerning the pharyngeal phase of swallowing [33]. Consequently, results of the patients investigated by HR-manometry were analyzed and also expressed in four severity levels despite the lack of related classifications. The lack of standardized classification algorithms is another limitation of our study. To overcome this limitation, findings were graded according to previously published surrogate parameters of motility disorders by two independent investigators. The Chicago Classification also describes different grades of disorders with regard to contraction vigor, and classivies major or minor disorders of peristalsis. We refrained from determining further thresholds for disorders, given the wide normal range of the values in HR-manometry and the fact that slight deviations often did not lead to impaired swallowing or rather have a clinical relevance [34]. In our analysis, results obtained by HR-manometry frequently differed from the results obtained by endoscopy (Fig.S2, Fig. S3, Fig.S4). Confirmatory, Cohen's kappa analysis showed poor agreement concerning the evaluation of the UES (Cohen's kappa coefficient: 0.141 ; SE of kappa 0.136). At least, the strength of agreement was fair referring to the assessment of tubular esophagus (Cohen's kappa coefficient: 0.323; SE of kappa 0.123). One of the reasons for the relatively low levels of strength of agreement was the chosen evaluation strategy: Putting complex results in four severity levels can lead to a certain degree of fuzziness. This concerns, in particular, our survey, that compares two fundamentally different diagnostic tools. Furthermore, borderline results are not represented by our analysis. In addition, like many other endoscopic examination methods, the technique is limited by operator dependency and variation in the evaluation of the swallowing function [35]. Moreover, swallowing function shows diurnal variations in terms of all swallowing phases [36]. In comparison to HR-manometry, which collects precisely measured data, functional endoscopy is much more dependent on subjective impressions of the underlying motility function.

\section{Conclusions}

In conclusion, functional endoscopy is a safe and effective diagnostic method for evaluation of patients suffering from neurogenic dysphagia. The novel feature of this technique is retroflexed visualization of the esophagus; by this means, the UES can be endoscopically evaluated for the first time. Furthermore, both tubular esophagus and LES can be monitored in the retroflexed position at functional endoscopy (observing the swallowing act after ingestion of solid food and liquid). Therefore, despite the limitations described above, the method can augment findings obtained with existing diagnostic tools, such as FEES and HR-manometry.

\section{Acknowledgements}

The authors thank Professor Gary Powell for helpful suggestions regarding the manuscript.

\section{Competing interests}

The authors declare that they have no conflict of interest.

\section{References}

[1] Dziweas R, Warnecke T. Neurogene Dysphagien: Diagnostik und Therapie. Kohlhammer Verlag; 2013

[2] Matsuo K, Palmer JB. Anatomy and physiology of feeding and swallowing: Normal and abnormal. Phys Med Rehabil Clin N Am 2008; 19 691-707

[3] Dziewas R, Glahn J, Helfer C et al. Flexible endoscopic evaluation of swallowing (FEES) for neurogenic dysphagia: Training curriculum of the German Society of Neurology and the German stroke society. BMC Medical Education 2016; 16: 70

[4] Martino R, Foley N, Bhogal S et al. Dysphagia after stroke: Incidence, diagnosis, and pulmonary complications. Stroke 2005; 36: 27562763

[5] Müller ], Wenning GK, Verny M et al. Progression of dysarthria and dysphagia in postmortem-confirmed Parkinsonian disorders. Arch Neurol 2001; 58: 259-264

[6] Morgan AS, Mackay LE. Causes and complications associated with swallowing disorders in traumatic brain injury. J Head Trauma Rehabil 1999; 14: 454-461

[7] Ates F, Vaezi MF. The Pathogenesis and Management of Achalasia: Current Status and Future Directions. Gut Liver 2015; 9: 449-463

[8] Wirth R, Dziewas R. Neurogene Dysphagie. Internist 2017; 58: 132140

[9] Prosiegel M. Neurogene Dysphagien. Leitlinien für Diagnostik und Therapie in der Neurologie. 2015 
[10] Farneti D, Consolmagno P. The Swallowing Centre: Rationale for a multidisciplinary management. Acta Otorhinolaryngologica Italica 2007; 27: 200-207

[11] Langmore SE, Schatz K, Olsen N. Fiberoptic endoscopic examination of swallowing safety: A new procedure. Dysphagia 1988; 2: 216-219

[12] Pandolfino JE, Fox MR, Bredenoord AJ et al. High-resolution manometry in clinical practice: Utilizing pressure topography to classify oesophageal motility abnormalities. Neurogastroenterol Motil 2009; 21: 796-806

[13] Baijens LW, Clavé P, Cras P et al. European Society for Swallowing Disorders - European Union Geriatric Medicine Society white paper: Oropharyngeal dysphagia as a geriatric syndrome. Clin Interv Aging 2016; 11: 1403-1428

[14] Bollschweiler E, Knoppe K, Wolfgarten E et al. Prevalence of dysphagia in patients with gastroesophageal reflux in Germany. Dysphagia 2008; 23: 172-176

[15] Herrmann IF, Recio SA. Functional pharyngoesophagoscopy: A new technique for diagnostics and analyzing deglutition. Oper Techniq Otolaryngol Head Neck Surg 1997; 8: 163-167

[16] Arens C, Herrmann IF, Rohrbach S et al. Positionspapier der DGHNO und der DGPP - Stand der klinischen und endoskopischen Diagnostik, Evaluation und Therapie von Schluckstörungen bei Kindern und Erwachsenen. Laryngorhinootologie 2015; 94: S306-S354

[17] Langmore SE. Endoscopic evaluation and treatment of swallowing disorders. New York: Thieme; 2001

[18] Pandolfino JE. High-resolution manometry: Is it better for detecting esophageal disease? Gastroenterol Hepatol 2010; 6: 632-634

[19] High-Resolution-Manometrie des Ösophagus: Bildatlas und Lehrbuch. Weingart V, Schönfeld A. Karlsruhe: Standard Light; 2012

[20] van Hoeij FB, Bredenoord AJ. Clinical application of esophageal highresolution manometry in the diagnosis of esophageal motility disorders. J Neurogastroenterol Motil 2016; 22: 6-13

[21] Kahrilas PJ. Esophageal motor disorders in terms of high-resolution esophageal pressure topography: What has changed? Am J Gastroenterol 2010; 105: 981-987

[22] Medtronic. Medtronic ManoScan ESO Brochure 2019: https://www. medwrench.com/documents/view/10314/medtronic-manoscaneso-brochure-pdf Accessed: 30.06.2020

[23] Ghosh SK, Pandolfino JE, Rice J et al. Impaired deglutitive EG] relaxation in clinical esophageal manometry: A quantitative analysis of 400 patients and 75 controls. Am J Physiol Gastrointest Liver Physiol 2007; 293: G878-G885

[24] Kahrilas PJ, Bredenoord AJ, Fox M et al. The Chicago Classification of esophageal motility disorders, v3.0. Neurogastroenterol Motil 2015; 27: $160-174$

[25] Carlson DA, Pandolfino JE. High-resolution manometry in clinical practice. Gastroenterol Hepatol 2015; 11: 374-384

[26] Bredenoord AJ, Fox M, Kahrilas PJ et al. Chicago classification criteria of esophageal motility disorders defined in high resolution esophageal pressure topography. Neurogastroentero Motil 2012; 24: 57-65

[27] Kuribayashi S, Iwakiri K, Kawada A et al. Variant parameter values-as defined by the Chicago Criteria-produced by ManoScan and a new system with Unisensor catheter. Neurogastroenterol Motil 2015; 27: 188-194

[28] Goetz CG, Poewe W, Rascol O et al. Movement Disorder Society Task Force report on the Hoehn and Yahr staging scale: Status and recommendations. Mov Disord 2004; 19: 1020-1028

[29] Rohof WOA, Bredenoord AJ. Chicago Classification of Esophageal Motility Disorders: Lessons Learned. Curr Gastroenterol Rep 2017; 19: 37

[30] Mielens JD, Hoffman MR, Ciucci MR et al. Automated analysis of pharyngeal pressure data obtained with high-resolution manometry. Dysphagia 2011; 26: 3-12

[31] Wang YT, Yazaki E, Sifrim D. High-resolution manometry: esophageal disorders not addressed by the "Chicago Classification". J Neurogastroenterol Motil 2012; 18: 365-372

[32] O'Sullivan SS, Massey LA, Williams DR et al. Clinical outcomes of progressive supranuclear palsy and multiple system atrophy. Brain 2008; 131: $1362-1372$

[33] Warnecke T, Oelenberg S, Teismann I et al. Endoscopic characteristics and levodopa responsiveness of swallowing function in progressive supranuclear palsy. Movement Disorders 2010; 25: 1239-1245

[34] Bredenoord AJ. Minor disorders of esophageal peristalsis: highly prevalent, minimally relevant? Clin Gastroenterol Hepatol 2015; 13: $1424-1425$

[35] Hoogenboom SA, Bagci U, Wallace MB. Al in gastroenterology. The current state of play and the potential. How will it affect our practice and when? Techniques in Gastrointestinal Endoscopy 2019: 150634

[36] Vaughn B, Rotolo S, Roth H. Circadian rhythm and sleep influences on digestive physiology and disorders. CPT 2014.67 